Revue de Défense nationale, Paris, été 2015

\title{
Le remplacement des F-16 belges : la dimension nucléaire
}

André Dumoulin ${ }^{1}$

Ces derniers mois, bien des commentaires ont été lancés autour du nucléaire militaire associé au remplacement des F-16 belges, dans les médias mais aussi lors d'auditions parlementaires. Des affrontements idéologiques ont actuellement lieu en Belgique sur fond de questionnements politiques, économiques et technologiques.

Certes, la question du nucléaire américain en Belgique est une vieille histoire, dès lors que durant la guerre froide, le pays était hôte de plusieurs types d'armes ${ }^{2}$ et pouvait les utiliser sur instruction et autorisation mécanique puis électronique, sous surveillance ${ }^{3}$ et déverrouillage américains - centralité d'autorité oblige - dans le cadre du principe général qu'était et qu'est toujours la dissuasion : éviter toute agression territoriale majeure visant les pays membres de l'Otan par la menace. Il s'agit actuellement pour la Belgique d'une capacité de larguer éventuellement des bombes accrochées (à l'unité) sous fuselage de certains de ses F-16, après décision américano-otanienne, avec possibilité pour le gouvernement belge de refuser la mission pour des motifs politiques. Ce que l'on appelle «la double clef» : seuls les ÉtatsUnis possèdent les codes d'activation des charges nucléaires, la Belgique fournissant le vecteur d'armes, en l'occurrence le F-16; chacun dépendant en quelque sorte de l'autre, nonobstant le fait qu'il existe d'autres bases nucléaires en Europe (Pays-Bas, Allemagne, Italie), y compris des bases nucléaires strictement américaines (Aviano, Incirlik).

Aujourd'hui, seules resteraient stockées des bombes thermonucléaires à gravité (puissance variable) ${ }^{4}$ sur la base de Kleine Brogel ${ }^{5}$. Le conditionnel est de mise dès lors que les contraintes juridiques bilatérales imposent de ne pas préciser le nombre ni leur localisation, des dépôts pouvant même être vides ou partiellement occupés. Cela explique le pourquoi du refus d'informer et de confirmer venant des autorités belges; violer les accords bilatéraux Belgique/États-Unis sur le haut secret nucléaire aboutirait à lancer des procédures judiciaires

\footnotetext{
${ }^{1}$ Attaché à l'Institut royal supérieur de défense (IRSD), professeur à l'Université de Liège.

${ }^{2}$ Mines de démolition, obus, ogives pour missile sol-sol et sol-air, bombes pour avions.

${ }^{3}$ La protection, la sécurisation, le transport et la petite maintenance seraient assurés par 130 militaires américains de la 52e Munition Support Squadron de l'USAF.

${ }^{4}$ Les modèles de bombes thermonucléaires américaines en Europe sont de type $B$ - 61 modèle 3 (puissances variables et réglables de 0,$3 ; 1,5 ; 60$ et $170 \mathrm{KT}$ ), modèle 4 (puissances de 0,$3 ; 1,5 ; 10$ et $45 \mathrm{KT}$ ).

${ }^{5}$ En dehors de Kleine Brogel (Belgique), les bases de Volkel (Pays-Bas), de Büchel (Allemagne), de GhediTorre et d'Aviano (Italie), et d'Incirlik (Turquie) seraient les autres bases nucléaires. Les deux dernières bases sont strictement américaines. Celle de Turquie n'accueille pas en permanence d'avions américains F-16.
} 
et des condamnations. Cela explique aussi concomitamment pourquoi certains hommes politiques belges qui critiquèrent la présence nucléaire dans le pays le firent... seulement lorsqu'ils quittèrent leurs fonctions ministérielles.

Tout le débat actuel repose à la fois sur la question de la modernisation ${ }^{6}$ des bombes B-61 vers le modèle 12 et surtout de leur association - obligatoire ou non - au nouveau appareil de combat F-35 (programme JSF).

D’ores et déjà, plusieurs questions se posent. Faut-il conserver une solidarité nucléaire transatlantique en maintenant la présence de quelque 10 à 20 charges nucléaires supposées à Kleine Brogel ? Cette question ne devrait trouver une réponse que dans un cadre collectif, faisant jouer la planification américaine, la bureaucratie otanienne, la notion de solidarité et le principe du consensus. Le curseur est aujourd'hui moins favorable au vu de la situation internationale, de la crise russo-ukrainienne, de la modernisation nucléaire russe et des incertitudes persistantes autour de la politique nucléaire iranienne ; ce qui ne prédispose pas à délivrer un message de désarmement nucléaire ou organiser un retrait unilatéral sans négociations multilatérales. En outre, pour la Belgique, pays hôte, accueillir du nucléaire américain serait une manière « de se faire bien voir », « de faire passer la pilule d'un budget de la défense nationale très réduit » à un moindre coût.

Une autre question est d'associer obligatoirement ou non le remplacement prévu des 54 avions de combat F-16 par un nouvel appareil avec l'emport de nucléaire. Ici se situe toute la symbolique du choix d'un nouveau vecteur d'armes. Entre l'Eurofighter européen, le Gripen suédois, le Rafale français ou le F-35 américain, les choix ne sont pas si ouverts que cela. Dans tous les cas, le choix sera politique, idéologique (appareil européen ou américain) et technologico-industriel pour les retombées souhaitées par les partis politiques formant la coalition gouvernementale ${ }^{7}$, mais aussi en tenant compte que le désarmement structurel opérera avec l'acquisition future d'un nombre plus réduit d'avions, crise économique et coût des appareils obligent.

Dès lors que le gouvernement suédois refuse que leur appareil vendu puisse être habilité à transporter des charges nucléaires et que l'Eurofighter est mal engagé dans la course commerciale vu ces performances mais aussi par la volonté allemande de ne pas accepter

\footnotetext{
${ }^{6}$ Un programme Life Extension Program (LEP) de modernisation des charges nucléaires est en cours de financement aux États-Unis, associant fiabilité et sécurité des sites. Les anciennes versions vont être rénovées/aménagées en version B-61 modèle 12 d'une puissance réglable avec un maximum autour de $50 \mathrm{KT}$ et d'une plus grande précision de ciblage (5-30 m) par l'intégration d'un système GPS et tir à distance de sécurité $(80 \mathrm{~km})$. Une mise en condition opérationnelle en Europe est prévue en 2024 autour de ce programme estimé à 9,6 milliards de dollars.

${ }_{7}$ Le parti socialiste francophone dans l'opposition a mis en avant toute l'importance des retombées économiques.
} 
l'intrusion américaine sur les informations technologiques sensibles de l'appareil construit en consortium afin d'y adjoindre des éléments de nucléarisation, les seuls appareils en lice seraient, au final, le Rafale et le F-35. Vaste dilemme, dès lors que le Rafale a acquis récemment les premières grandes ouvertures à l'exportation alors que les pilotes belges travaillent de concert à la fois avec les Américains, les alliés et les procédures Otan, les Hollandais voisins (F-16) et les Français. L'exigence nucléaire pourrait-elle s'avérer déterminante dans le choix belge ? Si la réponse est positive, un choix américain irait de soi. Si la réponse est négative, peut-on imaginer un choix belge qui « subtilement » reposerait sur un « désarmement par défaut » en choisissant un appareil de facto non nucléarisable ?

Peut-on imaginer le choix du Rafale qui impliquerait qu'Américains et Français soient en phase pour accepter de livrer «leurs secrets technologiques», y compris l'acceptation d'introduire des composants américains confidentiels et non accessibles associés au code d'activation, de contrôle et donc de liaison des charges nucléaires américaines, tout comme pour les Tornado italiens et allemands dont certains portent aujourd'hui la bombe B-61 ? Les garde-fous et les contraintes industrielles seraient légions et tout dépendrait de la position de Paris autant que de l'existence ou non d'un refus américain de perdre le marché belge du F35.

Sachant aussi que la position française non officielle prédisposerait probablement à ce que les Américains conservent une composante nucléaire de théâtre en Europe afin de ne pas être en première ligne avec les Britanniques si d'aventure Washington décidait de retirer leurs dernières bombes d'Europe au profit d'une stratégie de reconstitution visible et médiatisée en cas de crise nucléaire grave sur le Vieux Continent, tout comme elle le fait conventionnellement avec les transferts de chars Abrams dans l'Est de l'Europe ces dernières semaines.

Dans tous les cas, la France et le Royaume-Uni ont renforcé considérablement leur potentiel nucléaire en termes générationnels, avec de nouveaux calendriers capacitaires à l'horizon 2040. Une dénucléarisation hypothétique des quelque 10 à 20 (?) charges nucléaires stockées en Belgique n'aboutira aucunement à bouleverser le paysage nucléaire sur le Vieux continent. De toute évidence, le paysage nucléaire belge est à la fois incertain, sous surveillance et paradoxalement porteur de points de rupture. Parmi ceux-ci, l'hypothèse d'un paysage géostratégique européen totalement bouleversé, déserté nucléairement par les États-Unis, est difficile à imaginer aujourd'hui. Si cela devait apparaître, l'Hexagone deviendrait la seule puissance nucléaire disposant de vecteurs aériens nucléaires de théâtre en Europe de l'Ouest. 
La France aurait devant elle plusieurs pistes : celle du maintien centralisé de la posture nucléaire avec le maintien des appareils sur le sol républicain ; celle d'une ouverture intraeuropéenne avec l'hypothèse d'emploi de Rafale belges armés des missiles nucléaires francofrançais ASMP-A (pilotage mixte et double clef) parallèlement à la permanence de Rafale/ASMP-A proprement français; celle de la dispersion sur crise de certains Rafale/ASMP-A français sur quelques bases européennes hôtes de pays ayant assimilé historiquement la culture du nucléaire.

Ces différents paysages plausibles mais complexes et délicats diplomatiquement, seraient la résultante hypothétique, sinon utopique, d'une Europe toujours aux prises avec une Russie incertaine et une Amérique centralisant son nucléaire sur son territoire national. La permanence d'une dissuasion bien comprise serait alors nécessaire à horizon inchangé. Elle ne pourra alors qu'être franco-britannique ou associée à un noyau dur de quelques pays européens qui veulent aller «plus vite et plus loin» et qu'appellent de leurs vœux les européistes et les plus fédéralistes ${ }^{8}$ sous l'expression d' «Union européenne de sécurité et de défense ».

Entre diplomatie, géopolitique, doctrine, intérêts vitaux, solidarité d'alliance, perception des menaces, aspects industriels et budgétaires, éthique de responsabilité et principes de précaution, le dossier nucléaire est tout, sauf simple. Le dossier du remplacement des F-16 belges a véritablement fait revenir le sujet « par la fenêtre ». Le Plan stratégique belge retardé mais qui doit aboutir à des clarifications cette année encore pourrait réduire le nombre d'hypothèses et effacer ou confirmer certaines audaces ; nonobstant le fait que dans ce pays complexe et surréaliste, la position gouvernementale semble différente de celle de représentants de partis politiques qui font partie de la même coalition au pouvoir !

\footnotetext{
${ }^{8}$ André Dumoulin : «Armée européenne : quelle phase transitoire ?, », Europe Diplomatie \& Défense, Agence Europe, Bruxelles, 7 mai 2015.
} 